70. Tobagoa maleolens Urb., spec. nov.

Planta 0,6-1 m alta suffrutescens maleolens (ex Egg. ot Broadw.). $\mathrm{R}$ ami lineis 4 prominentibus e basi petiolorum decurrentibus notati, inter lineas binas magis approximatas applanati v. concaviusculi, inter binas remotiores convexi, glabri laeves. Stip ularum vagina $0,5-1 \mathrm{~mm}$ longa, setis 3 inter sese remotis apice plerumque calloso-incrassatis, $2-4 \mathrm{~mm}$ longis. Folia 3-8 $\mathrm{mm}$ longe petiolata, basi sensim in petiolum angustata, antice longe et acutissime acuminata, majora $7-10 \mathrm{~cm}$ longa, inferne v. sub medio latissima, $2-3 \mathrm{~cm}$ lata, nervo medio supra inferne anguste impresso, lateralibus utroque latere $5-6$ sub angulo $30-40^{\circ}$ abeuntibus, subtus flavo-albescentibus, non anastomosantibus, supra pilis perbrevibus scabrida, subtus brevissime pilosa, membranacea plana. Capitula in axillis folii utriusque obvia 10-20-flora; bracteae tenuissime membranaceae e cellulis strue unica dispositis contextae, ratione latissimae, ambitu semiorbiculares, a medio v, paullo profundius inaequaliter lacerae, laciniis $v a l d e$ inaequilongis linearibus $v$. lanceolatis, margine pilosis. $\mathrm{CaJ} \mathrm{yc}^{\mathrm{c}}$ is lobi subaequales $0,5-0,6 \mathrm{~mm}$ longi. Corolla $3 \mathrm{~mm}$ longa; lobi in flor. dolichostylis ovato-lanceolati tubo dimidio longiores, in flor. brachystylis triangulari-ovati tubo dimidio breviores, obtusiusculi. Filamenta in flor. dolich. tubo corollae sub sinubus inserta, $0,4 \mathrm{~mm}$ longa, in thor. brachyst. tubo inter lobos in sinubus affixa $1 \mathrm{~mm}$ longa; antherae clausae ovalioblongae, effoetae oblongo-lineares, apice subtruncatae, $0,8 \mathrm{~mm}$ longae. Stylus in flor. dolich. $4 \mathrm{~mm}$ longus, in $1 / \mathrm{s}$ superiore bifidus, in brachyst. fere $2 \mathrm{~mm}$ longus, in $1 / 4$ superiore bifldus. Capsula brevissime stipitata, circumcirca brevissime pilosa, 1,5 mm longa. Semina vix supra $1 \mathrm{~mm}$ longa, $0,6 \mathrm{~mm}$ lata, nigrescentia.

Hab. in Tobago in convalli superiore fluminis Great Dog river, m. Nov. flor.: Eggers no. 5783 (typus), in sylvis ad Belmont, m. Mart. flor.: Broadway no. 3527, prope Charlotte ville ad Pigeon Hill, m. April. flor.: Broadway no. 3629 .

\title{
LIX. Eine neue Art der Leguminosen-Gattung Leptoderris Dunn aus Kamerun.
}

Von H. Harms.

(Originaldiagnose.)

Leptoderris tomentella Harms, spec. nov.

Frutex scandens, ramulis brevissime incano-tomentellis; folia imparipinnata, petiolata (petiolo $3-6 \mathrm{~cm}$ longo), rhachi cum petiolo breviter tomentella, 4-10 cm longa, foliola bijuga, breviter $(5-7 \mathrm{~mm})$ petiolulata (petiolulis crassiusculis), obovato-oblonga vel obovata vel oblonga, basi angusta vel latiuscula obtusa vel rotundata vel emarginulata, apice obtusa vel rotundata vel brevissime acuminulata vel emarginata (lateralia 土 obliqua), subcoriacea vel coriacea, supra nitidula (juvenilio brevissime pul- 
verulento-tomentella), subtus dense requaliter brevissime tomentella nervis lateralibus paucis (3-4) ascendentibus cum nervo medio subtus prominulis, 4-12 cm longa, 2,5-8 cm lata; stipellue brevissimae gibberi formes; paniculae terminales amplae multiflorae, brevissime tomentellae. ramulis racemiformibus elongatis angustis $7-20 \mathrm{~cm}$ longis, flores brevissime $(1-2 \mathrm{~mm})$ pedicellati, in glomerulos paucifloros ad nodos ramulorum brevissimos pulviniformes dispositi, 9-10 mm longi; calyx anguste oblique cupuliformis, breviter late denticulatus tomentellus, 3-4 mm longus, basi bracteolis geminis minimis ovatis vel suborbicularibus instructus; corolla glabra exserta, in sicco atropurpurea; ovarium pubescens.

Kamerun: Bipinde, Mimfia-Berg, Niederung am Südhang (Zenker no. 4942, Mai 1913; Blätter oben grün, unten silbergraubraun-wollig, Blüten schmutzig weiss).

$L$. tomentella gehört nach der Bestimmungstabelle von Dunn (Kew Bull. .[1910] 388) in die Nähe von $L$. micrantha Dunn und $L$. reticulata Dunn (beide von Lagos), und dürfte besonders der letzteren nahekommen, von der sie sich durch geringere Zahl der Seitennerven der Blättchen, gleichmässige kurzfilzige Behuarung der Blattunterseite und kleinere Blïten mit trocken schwärzlicher Krone unterscheidet. Sehr nahe steht unserer Art wohl auch $L$. velutina Dunn in Kow Bull.(1914), 246 (Franz. Kongo), bei der aber die Nerven der Blättchen 6-paarig sein sollen; ausserdem solien bei $L$. velutina die Stipellen $3-4 \mathrm{~mm}$ lang sein, während sie bei unserer Art ganz kurz sind.

\section{Vermischte neue Diagnosen.}

1025. Rulac Nuttallil Nieuwland in Amer. Midl. Nat., II (1911), p. 137. - Negundo or Acer fraxinifolium Nuttall, 1818, not Negundium fraxinifolium Raf., 1808. - Small or middle sized tree, with white or bluish glaucous twigs whose bark is usually somewhat thicker than in the preceding; leaves pinnately or incompletely bipinnately compound; leaflets usually smaller than those of the preceding, 3-11, usually $3-7$ on fruiting branches and 5-11 on sterile shoots, leaflets rather thick and veiny, dark-green above and paler beneath, glabrous on both sides except on the veins of the lower surface, with rather densely pubescent tufts in the angles of the principal veins; leaflets variously and irregularly toothed and cleft especially the terminal and basal lateral which are often again trifoliolate; secondary veins prominent and mesophyl pale beneath; flowering and fruiting racemes usually 4 on a twig, opposite in pairs, with numerous samaras, and at the ends of the twigs of preceding years growth; racemes on short side branches $1 \mathrm{~cm}$ or less in length, with or without reduced simple or ternate leaves; peduncles 2-2,5 cm long: samaras densely clustered, in appearance usually smaller: samara wings joined usually at an acute angle at the base, and not at all, or not much attenuated; fruit body glabrous. - From Rulac interior it is easily distinguished by its glaucous white twigs, those of 